\title{
Enhanced recovery after surgery in intramedullary and extramedullary spinal cord lesions: perioperative considerations and recommendations
}

\author{
Sauson Soldozy $\mathbb{1}^{1} \cdot$ Parantap Patel $^{1} \cdot$ Mazin Elsarrag ${ }^{1} \cdot$ Pedro Norat $^{1} \cdot$ Daniel M. Raper $^{1} \cdot$ Jennifer D. Sokolowski ${ }^{1} \cdot$ \\ Kaan Yağmurlu $\mathbb{D}^{1} \cdot$ Min S. Park ${ }^{1} \cdot$ Petr Tvrdik $^{1} \cdot$ M. Yashar S. Kalani ${ }^{1}$
}

Received: 6 January 2019 / Revised: 8 July 2019 / Accepted: 9 July 2019 / Published online: 29 July 2019

(c) The Author(s), under exclusive licence to International Spinal Cord Society 2019

\begin{abstract}
Enhanced recovery after surgery (ERAS) is an evidence-based approach developed to ameliorate the patient recovery process following surgical procedures. Employing a multimodal, multidisciplinary approach, ERAS implements strategies and treatment paradigms that have been shown to improve patient outcomes, reduce hospital length of stay, and ultimately reduce healthcare costs. With a substantial body of the literature supporting the implementation of ERAS in other surgical specialties, ERAS has only recently made its foray into spine surgery. Despite this, current studies are limited to spinal deformity and degenerative disease, with limited data regarding spinal cord surgery. This is due in part to the complex nature and rarity of spinal cord lesions, making the establishment of a formal ERAS protocol difficult. In developing an ERAS protocol, there must be a consensus on what factors are important to consider and implement. To address this, we reviewed the most recent advances in intramedullary and extramedullary spinal cord surgery in order to identify elements that influence patient outcomes. Using this information, the authors provide evidence-based recommendations with the intent of introducing a framework for future ERAS protocols with respect to treating spinal cord lesions.
\end{abstract}

\section{Introduction}

Enhanced recovery after surgery (ERAS) is a multidisciplinary, multimodal approach to improving surgical outcomes using subspecialty- and procedure-specific evidence-based protocols in the care of surgical patients [1]. Originally formalized by a collaborative group of European surgeons in 2001, ERAS adheres to four key principles: (1) the use of a multidisciplinary team to work harmoniously in the care of a single patient, (2) the use of a multimodal approach to resolve issues inhibiting the recovery process, (3) the use of evidence-based guidelines in patient care, and (4) the implementation of a continuous audit to ensure program adherence and improve outcomes [1]. Initially described as "fast track surgery," systematic approaches to perioperative care were espoused in the

M. Yashar S. Kalani

stemcelldoctor@kalanimed.com

1 Department of Neurological Surgery, University of Virginia Health System, Charlottesville, VA, USA literature as early as the 1990s in the setting of cardiopulmonary bypass surgery [2].

Given the apparent benefits of ERAS programs in other surgical disciplines its increasing implementation in spine surgery is not surprising. Spinal procedures are associated with high rates of postoperative pain and morbidity, slow return to function, and prolonged hospital stays [3]. It therefore follows that ERAS may have similar benefits in spine surgery patients. An evidence-based platform intended to streamline care and reduce cost burden, ERAS has the potential to facilitate quicker return to function and alleviate financial strain for patients undergoing spinal cord surgery. To date, there have been no ERAS protocols developed for spinal cord surgery. This is primarily due to a lack of standardized treatment paradigms and guidelines in this specific cohort of patients. In this study, the authors review the current literature regarding intramedullary and extramedullary spinal cord surgery. Focusing on the treatment of cavernous malformations (CMS), spinal vascular malformations, ependymomas, and astrocytomas, different treatment approaches are considered, and evidence-based recommendations, based on the American Heart Association guidelines, are made regarding improved patient 
outcomes and potential utility in the development of ERAS protocols in spinal cord surgery (Table 1).

\section{Spinal cavernous malformations}

CMs of the spine are vascular lesions that can present as intramedullary [4], intradural extramedullary [5], extradural intraspinal [6], and extradural with paraspinal extension [7]. Classified as a capillary-venous pathology, CMs are characterized by endothelial-lined low-flow vascular channels surrounded by hemosiderin rings without parenchymal involvement [8]. CMs are benign lesions that occur in $0.5 \%$ of the general population and are most often found incidentally on magnetic resonance imaging (MRI) [8], with familial cases often presenting with multiple intracranial and intraspinal lesions (Fig. 1a-d). Symptomatic spinal cord CMs are a result of hemorrhage, with an estimated bleeding risk between 1.4 and $4.5 \%$ per year [9]. Patients with an acute hemorrhage will present with symptoms of spinal pain or radiculopathy corresponding to the level of the lesion, occurring most commonly in the thoracic spine [4]. Cases of gradual neurologic dysfunction are suggestive of chronic myelopathy and may resemble demyelinating disorders [10]. Given the infrequent incidence of spinal CMs, a formal ERAS protocol has yet to be developed; despite this, perioperative considerations are discussed along with their potential utility in an ERAS protocol.

\section{Preoperative period}

Preadmission counseling is a key component of ERAS in the preoperative period as it sets the tone for the remainder of the patient encounter. When considering patients for cavernoma resection, the risks of intervention must be weighed against the risk of hemorrhage (1.4-4.5\% per year) [11]. Asymptomatic patients found to have incidental lesions are recommended MRI and clinical monitoring. When intervention is indicated, patients and their families should be informed of the possible risk of transient neurological worsening, which is to be expected when resecting CMs in eloquent regions. As is the case for cranial CMs, acute worsening may resolve [12], although postoperative rehabilitation may be necessary. A recent series reported in a cohort of 83 patients that $9.6 \%$ of patients worsened, $68.7 \%$ were unchanged, and $21.7 \%$ of patients experienced improvement immediately after surgery [13]. Candidates of surgery should also be advised against waiting for treatment, as surgical timing has been implicated in favorable postoperative outcomes. A positive correlation between operating within 3 months of symptom onset and favorable clinical outcome (OR 2.11, 95\%CI 1.31-3.41, $p=$
0.002) was recently reported [9]. Furthermore, it should be explained to patients that while resection effectively reduces pain, functional deficits secondary to chronic myelopathy are less likely to reverse, with surgical resection merely halting disease progression in these patients [9].

\section{Intraoperative period}

Intraoperative considerations include monitoring of spinal somatosensory evoked potentials and motor evoked potentials, which play a role in enhancing patient recovery [14]. In addition, the monitoring and maintenance of mean arterial pressure (MAP) at $85 \mathrm{mmHg}$, which is based on traumatic spinal cord injury (SCI) guidelines, may help to avoid spinal cord ischemia [15]. Ultrasound mediated image guidance is another intraoperative tool available for neurosurgeons. First utilized for CM resection in 1994, intraoperative ultrasonography can aid in visualizing CM lesions that present without an exophytic component [16]. This enables localization of the exact site for myelotomy as well as to provide confirmation of total gross resection, especially in deep-seated lesions [17]. The neurosurgeon should aim for gross total resection (GTR) (Fig. 1e), as it is a statistically significant predictor of good outcome (OR $3.61,95 \%$ CI 1.24-10.52, $p=0.02$ ) (class I; level of evidence: C) [9]. Recurrence rates of up to $5 \%$ have been reported in cerebral $\mathrm{CMs}$ and are often attributed to residual cavernoma that was not detected. With most lesions presenting dorsally, a posterior approach with osteoplastic laminoplasty or keyhole laminotomy is preferred. When possible, a minimally invasive approach should be employed. A recent meta-analysis stated that hemilaminectomy was associated with better outcomes than total laminectomy or laminoplasty (OR 3.20, 95\% CI $1.16-8.86, p=0.03)$, resulting in a reduction of complications related to vertebral column instability and iatrogenic SCI [9]. When resecting the lesion, en bloc excision is preferred, but piecemeal removal may be necessary for debulking of larger lesions to reduce SCI [18]. Resection of the hemosiderin fringe is not recommended given that its resection would injure eloquent tissue [19]. Surrounding cryptic venous malformations should also be preserved, otherwise significant deficits can occur as these venous anomalies often serve as the primary drainage site for adjacent tissues [20]. An alternative option to traditional microsurgical techniques involves $\mathrm{CO}_{2}$ laser resection, which has been reported to cause less edema and thermal damage in comparison with bipolar cautery [21]. The advantage of the OmniGuide $\mathrm{CO}_{2}$ laser (Cambridge, MA) lies in its ability to reach narrow surgical corridors and was reported to increase complete resection rates with less injury to adjacent tissue structures [22]. 
Table 1 Descriptive overview of studies describing spinal cord tumor surgery

\begin{tabular}{|c|c|c|}
\hline Study & Focus of study & Principle relevant conclusions \\
\hline \multicolumn{3}{|l|}{ Spinal cavernous malformations } \\
\hline $\begin{array}{l}\text { Sandalcioglu et al. [11], Zevgaridis } \\
\text { et al. [56] }\end{array}$ & Risks of untreated lesion & $1.4-4.5 \%$ risk of hemorrhage per year if untreated \\
\hline Walcott et al. [12] & Outcomes of surgery & Acute post-op worsening of function may resolve or may require rehabilitation \\
\hline Li et al. [13] & Outcomes of surgery & $9.6 \%$ worsened function, $68.7 \%$ unchanged, $21.7 \%$ improved $(N=83)$ \\
\hline \multirow[t]{2}{*}{ Badhiwala et al. [9], Kim et al. [54] } & Outcomes of surgery & $\begin{array}{l}27.2 \% \text { experienced early transient post-op complications. Positive correlation between } \\
\text { operating within } 3 \text { months of symptoms and favorable outcomes }\end{array}$ \\
\hline & & Deficits from chronic myelopathy less likely to reverse \\
\hline Mitha et al. [23], Jallo et al. [53] & Outcomes of surgery & $10.7 \%$ worsened function, $37.8 \%$ unchanged, $51.5 \%$ improved $(N=?)$ \\
\hline Badhiwala et al. [9], Bian et al. [51] & Outcomes of surgery & $\begin{array}{l}\text { GTR is predictor of good outcomes. Hemilaminectomy associated with better outcomes } \\
\text { versus total laminectomy/laminoplasty }\end{array}$ \\
\hline Muramoto et al. [14] & Intraoperative monitoring & Use of intraoperative evoked potential monitoring improves patient recovery \\
\hline Lunardi et al. [16] & Intraoperative monitoring & Use of intraoperative ultrasound imaging improves visualization \\
\hline Vishteh et al. [18] & Surgical technique & Piecemeal removal for large lesions is recommended \\
\hline Deshmukh et al. [19] & Surgical technique & Avoid resection of hemosiderin fringe \\
\hline Vishteh et al. [20] & Surgical technique & Preserve peri-lesion cryptic venous malformations to avoid extended tissue damage \\
\hline Ryan et al. [21], Choudhri et al. [22] & Surgical technique & $\mathrm{CO} 2$ laser resection may cause less edema and thermal damage versus bipolar cautery \\
\hline \multicolumn{3}{|l|}{ Spinal vascular malformations } \\
\hline Rangel-Castilla et al. [32] & Outcomes of surgery & $\begin{array}{l}\text { Transient neurologic decline in } 6.8 \% \text { with AVFs and } 30.3 \% \text { with AVMs; } 71.4 \% \text { of these } \\
\text { with AVFs and } 43.6 \% \text { of these with AVMs show improvement at average } 30.5 \text { months } \\
\text { follow-up }(N=110)\end{array}$ \\
\hline $\begin{array}{l}\text { Wojciechowski et al. [33], Yue et al. } \\
\text { [42] }\end{array}$ & Outcome of surgery & $\begin{array}{l}\text { More severe pre-op presentation associated with minimal benefit from surgery and greater } \\
\text { LOS, although symptoms likely to be stabilized with surgery }\end{array}$ \\
\hline Yue et al. [42] & Costs of surgery & Complications from surgery increase cost by US $\$ 30,000$ \\
\hline Cenzato et al. [34] & Outcome of surgery & Motor deficits more likely to improve versus sensory and sphincter disturbances \\
\hline $\begin{array}{l}\text { Wojciechowski et al. [33], Cenzato } \\
\text { et al. [34] }\end{array}$ & Outcome of surgery & Timing of surgery relative to symptoms onset not predictive of outcome \\
\hline Ozpeynirci et al. [36] & Intraoperative monitoring & 3D-RA enhances visualization of lesion \\
\hline Kang et al. [37] & Outcomes of surgery & Surgical resection associated with less recurrence versus embolization $(N=26)$ \\
\hline Česák et al. [38] & Outcomes of surgery & Surgical resection associated with less recurrence versus embolization $(N=24)$ \\
\hline Gokhale et al. [39] & Surgical technique & Liquid embolic agents associated with less recurrence versus particle embolization \\
\hline \multicolumn{3}{|l|}{ Intramedullary spinal cord tumors } \\
\hline Grasu et al. [3] & Outcomes of ERAS & $\begin{array}{l}\text { Formulated ERAS protocol showed decreased LOS, 30-day readmission, 30-day } \\
\text { complications, and pain scores versus control protocol, although not statistically } \\
\text { significant in setting of metastatic lesions }(N=97)\end{array}$ \\
\hline \multicolumn{3}{|l|}{ Ependymomas } \\
\hline Tobin et al. [43], Kucia et al. [44] & Outcomes of surgery & GTR is feasible and first line of treatment (class I, evidence level C) \\
\hline Feldman et al. [45], Guidetti et al. [46] & Outcomes of surgery & $\begin{array}{l}\text { GTR is strong predictor of survival, but not functional outcome. STR results in higher } \\
\text { recurrence }\end{array}$ \\
\hline Tobin et al. [43] & Management & $\begin{array}{l}\text { Adjuvant radiotherapy is second line of treatment for STR (class IIa, evidence level C). } \\
\text { Chemotherapy is second line of treatment for STR in children (class IIb, evidence level C) }\end{array}$ \\
\hline Fujiwara et al. [52], Kim W-H et al. [55] & Management & Temozolomide effective as salvage therapy of myxopapillary ependymomas \\
\hline \multicolumn{3}{|l|}{ Astrocytomas } \\
\hline Tobin et al. [43], Mottl et al. [50] & Outcomes of surgery & $\begin{array}{l}\text { Surgery is first line of treatment (class IIb, evidence level C). STR with adjuvant } \\
\text { radiotherapy (class IIa, evidence level C) or chemotherapy (class IIb, evidence level C) for } \\
\text { high grade tumors }\end{array}$ \\
\hline Teng et al. [47] & Management & Temozolomide promising for treatment \\
\hline
\end{tabular}

Table 1. Summaries of studies categorized by pathology

GTR gross total resection, $A V M s$ arteriovenous malformations, $A V F s$ arteriovenous fistulas, $L O S$ length of stay, $3 D-R A$ three-dimensional rotational angiography, $S T R$ subtotal resection

\section{Postoperative period}

A meta-analysis including 632 total patients reported that early transient postoperative complications occur at a rate of $27.2 \%$ [9]; this includes but is not limited to deep vein thrombosis, cerebral spinal fluid leak, or serous fluid collection. It was also reported that on follow-up, neurological function was worse in $10.7 \%$ of patients, unchanged in $37.8 \%$, and better in $51.5 \%$ of patients [23]. Sensory symptoms, such as pain and paresthesias, are more likely to improve than motor deficits [24].

An extensive postoperative rehabilitation process is necessary based on the neurological symptoms that persist after surgery. When compared with patients with traumatic 
Fig. 1 A 58-year-old lady presented with incontinence, imbalance, and bilateral painful upper extremity paresthesiae, and was found to have multiple intracranial and intraspinal lesions consistent with cavernomas (a, b T2 sagittal and axial; c, d T1 postcontrast sagittal and axial). Genetic testing identified a heterozygous pathogenic variant in the KRIT1 gene associated with autosomal dominant CCM. She underwent C4-C6 laminectomies and resection of the large, symptomatic cervical cavernoma. Postoperative imaging revealed resection of the lesion with reduction in surrounding cord signal change (e)
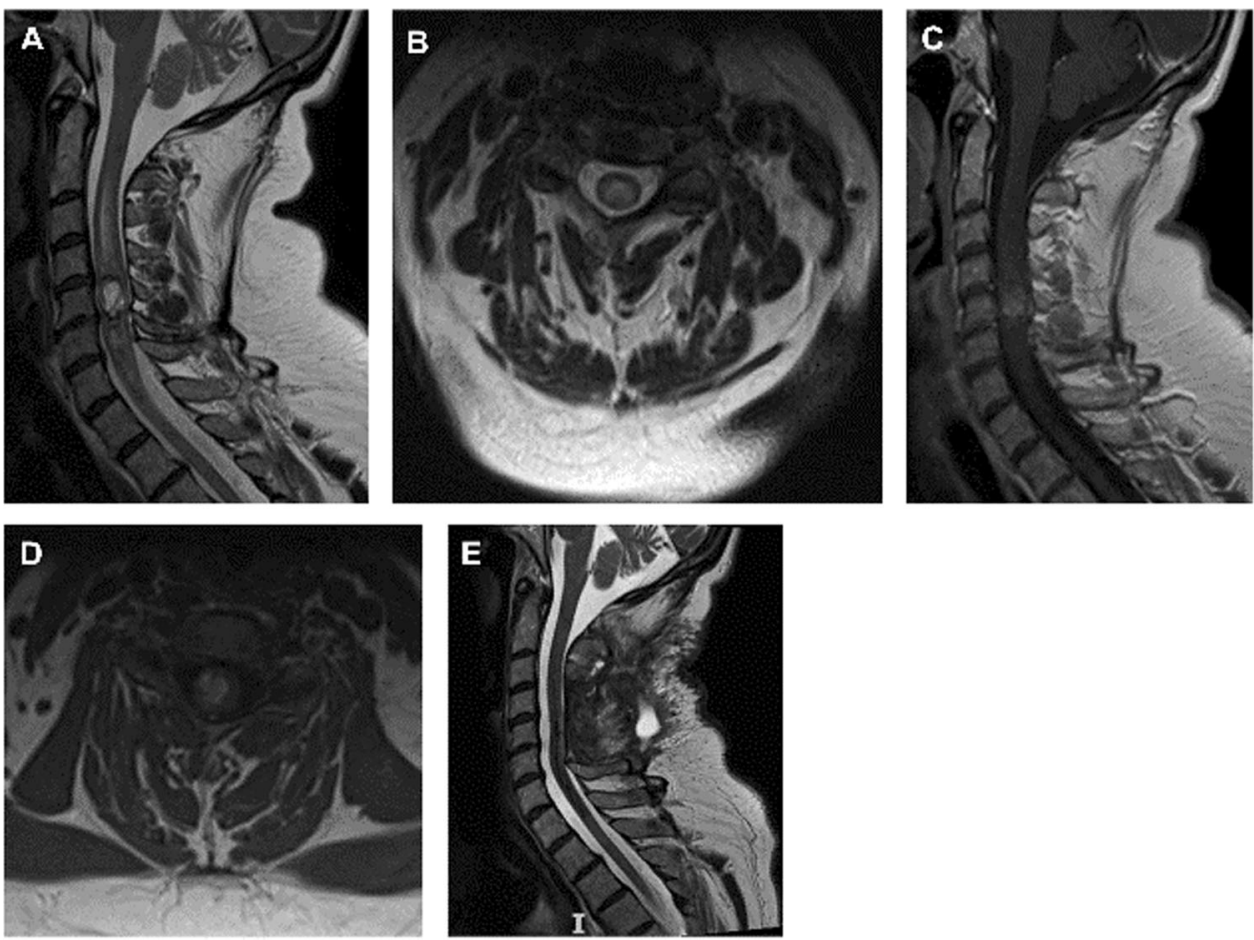

SCI, patients with neurological deficits due to neoplasm have a decreased length of stay of inpatient rehabilitation and should be approached differently given the incomplete nature of injury [25]. The most improved neurological recoveries are seen in patients with benign tumors. In addition to rehabilitation, the use of incentive spirometry, optimal nutritional supplementation, mood management, and skin care education has been shown to contribute to improved survival in the 20-week period [26].

\section{Recommendations}

Based on the present literature, the authors recommend that an ERAS protocol for CMs should place a large emphasis on patient education so that realistic expectations can be established for the immediate and long-term postoperative period. Specifically, the protocol must ensure that all healthcare team members be able to explain that treatment may not resolve their symptomology, and that functional decline may likely present with greater deficits than were initially present following surgery. This helps to deter distrust and patient dissatisfaction, better preparing patients for surgery and the rehabilitation process afterwards. The necessity of early intervention should be stressed to patients, since it is a positive predictor of improved outcomes. For the intraoperative period, the present literature suggests a minimally invasive keyhole approach is associated with better outcomes. In addition, standard neurosurgical monitoring should always be employed, including maintenance of MAP at $85 \mathrm{~mm} \mathrm{Hg}$. We recommend GTR with sparing of the hemosiderin ring and cryptic malformations, while avoiding piecemeal resection when possible. The authors also present the option of $\mathrm{CO}_{2}$ laser resection in conjunction with standard techniques to reduce the risk of swelling and edema. In the postoperative period, transient worsening is to be expected and, depending on the patient's baseline neurologic status prior to surgery, there may be persistent dysfunction indicative of SCI. For this reason, an extensive postoperative rehabilitation process utilizing both physical and occupational therapy is necessary. As further advancements are made with respect to the treatment of spinal CMs, more evidence-based improvements can eventually be integrated into a formal ERAS protocol.

\section{Spinal vascular malformations}

Spinal arteriovenous fistulas (AVFs) and arteriovenous malformations (AMVs) are complexed lesions that are categorized based on the anatomical location and corresponding pathophysiological mechanism. This includes extradural AVFs, dorsal intradural AVFs, ventral intradural AVFs, extradural-intradural AVMs, intramedullary AVMs, and conus medullaris AVMs [27]. Dural AVFs account for $70 \%$ of spinal vascular malformations, making it the most common lesion subtype, with spinal AVMs being the second most common at $20 \%$ [28, 29]. While motor and sensory deficits, incontinence, and pain are shared manifestations of both spinal AVFs and AVMs [30], they 

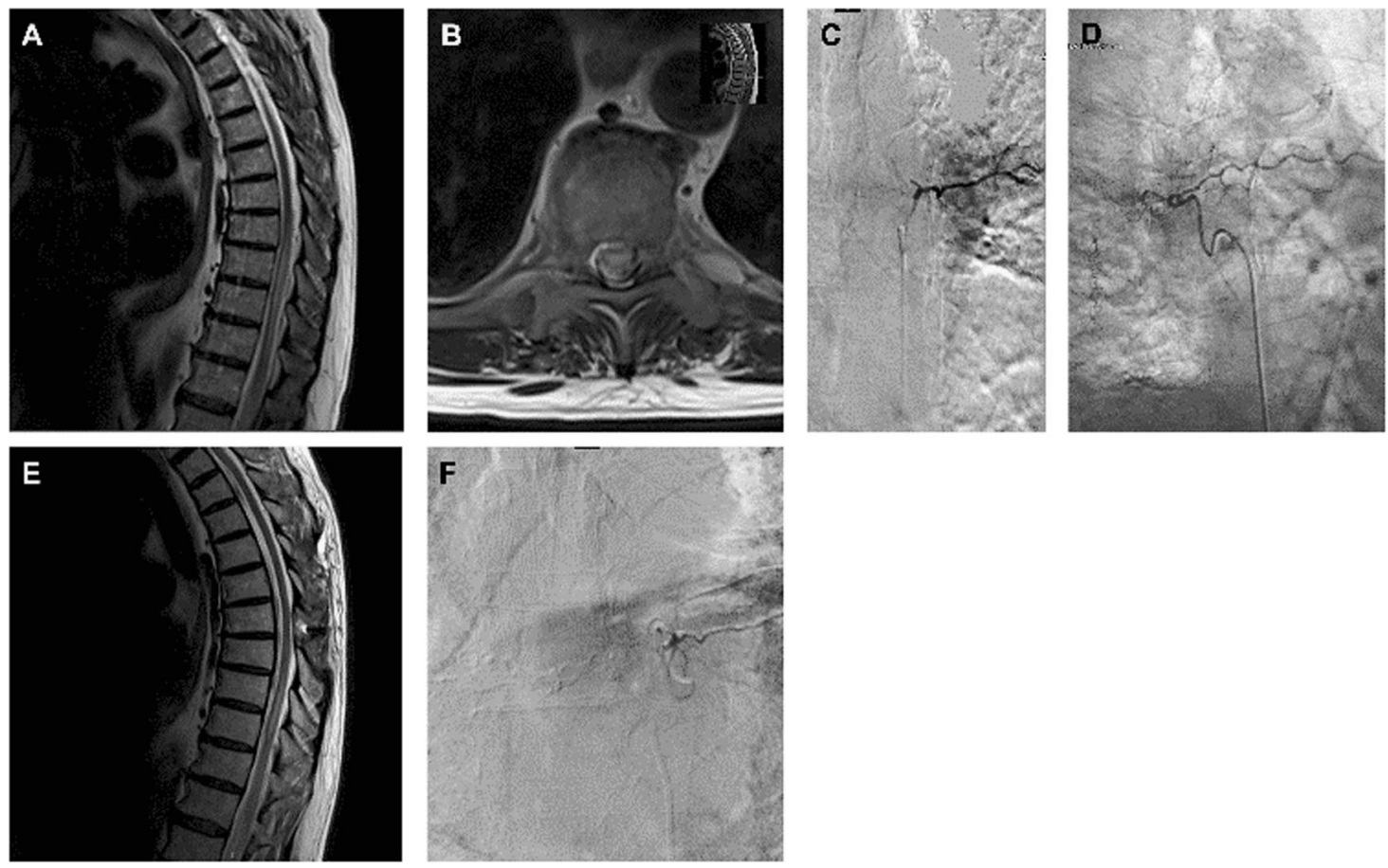

Fig. 2 An 82-year-old man presented with a subacute history of progressive lower extremity weakness, sensory changes, and bladder incontinence. Spinal MR and MRA revealed signal change in the lower thoracic cord, with multiple prominent enhancing perimedullary intradural veins in the mid and lower thoracic spine suggestive of a dural arteriovenous fistula (dAVF) (a, b). Spinal angiography revealed a Type 1 spinal dAVF supplied by the radicular branch of the left T7 intercostal artery. The fistula drained into a tortuous dorsal venous complex which slowly descends to the conus. The Artery of

can be differentiated by their unique symptomology and presentation. Thoracolumbar pain with or without radiculopathy in conjunction with chronic progressive sensorimotor impairment and incontinence is characteristic of spinal AVFs, with spinal MR and MRA confirming diagnosis (Fig. 2a, b). Subarachnoid hemorrhage is extremely rare as they are low-flow lesions. This contrasts with spinal AVMs, which present more acutely with either upper or lower extremity symptoms due to hemorrhage, mass effect, or vascular steal exacerbated by physical exertion [30]. Hemorrhage occurs in spinal AVM patients at an annual rate of $4 \%$, increasing to $10 \%$ following an initial bleed [31]. Given the relative rarity of spinal vascular malformations, treatments are based on the retrospective series. As is the case with CMs, there lacks an established ERAS protocol, although important observations have recently been made with respect to optimizing clinical outcomes that can help establish the basis for a formal paradigm.

\section{Preoperative period}

Improving patient satisfaction begins at the preadmission stage with ensuring understanding of the disease process
Adamkiewicz was visualized arising from the left T8 intercostal artery. Coil embolization for preoperative level marking was performed at the left T7 level. The patient was taken the following day for a T7 laminoplasty and surgical ligation of the dAVF. The patient made a good recovery and was discharged without operative complication. Followup spinal angiogram performed at 3 months demonstrated no residual dAVF (e). MRI performed 6 months after surgical ligation demonstrated stable cord signal change with reduced venous engorgement (f)

and establishing reasonable expectations following intervention. Like cavernomas, patients should be informed that it is possible they will experience no change in symptoms or even transient worsening immediately following intervention, although this may resolve on follow-up and is dependent on the specific subtype of spinal vascular malformation present. Patients with AVFs are more likely to experience worsening following treatment, although most will exhibit overall improvement. In a series of 110 patients (44 with AVFs and 66 with AVMs), transient neurologic decline was observed in 6.8 and $30.3 \%$ of patients with spinal AVFs and AVMs, respectively. In patients who had postoperative decline, $71.4 \%$ of patients with AVFs and $43.6 \%$ of patients with AVMs showed improvements at 30.5 months mean follow-up. In patients with spinal AVMs, lesion site was a factor in functional recovery, with conus medullaris AVMs having the best recovery and extradural lesions being the worst $(68.7 \%$ vs $20 \%)$ [32]. Patients with severe clinical presentations should also be counseled accordingly. It is important to elucidate the relationship between preoperative symptomatic severity and neurologic outcome, with more severe preoperative presentations being associated with minimal benefit from intervention and 
greater hospital length of stay, although symptom stabilization is likely to be achieved [33]. Unlike CMs, timing of surgery relative to symptom onset is not predictive of functional outcome [34].

\section{Intraoperative period}

Establishing a treatment paradigm for spinal vascular malformations is difficult. Depending on the lesion, a multimodal approach utilizing both endovascular embolization and surgical resection may be necessary, with treatment options being tailored specifically to the patient's unique clinical presentation and angioarchitecture [35]. Recently, three-dimensional rotational angiography (3D-RA) has been used in conjunction with conventional biplane projections, enhancing visualization and aiding in the treatment of complex spinal dural AVFs [36]. In the treatment of spinal intradural dorsal AVFs, one series reported that the recurrence rate of 14 patients who underwent embolization only was $36 \%$, whereas none of the 12 patients who underwent surgical resection experienced recurrence [37]. Another series of 24 spinal intradural AVFs (11 endovascular and 13 surgical) reported similar results, with recurrence occurring in $35.3 \%$ of patients in the endovascular group and zero recurrence in the surgical group [38]. Less recurrence is noted with liquid embolic agents, such as n-butyl cyanoacrylate (nBCA) and Onyx, with the use of particle embolization being contraindicated [39]. Prior to embolization, provocative testing can be performed to identify functionally eloquent territories where pathologic vasculature may be difficult to distinguish from normal vasculature. If the provocative test is positive, embolization should not be performed at the given catheter position, suggesting advancement closer to the nidus or embolization from an alternative feeder is necessary [40]. The degree of surgical resection is contingent upon the extent of invasion with respect to the feeding arteries and draining veins of the nidus. Used preoperatively, embolization can also be utilized to provide vascular access to lesions and disconnection at the fistula site, aiding in surgical resection (Fig. 2c, d). Intramedullary AVMs with large diving vessel loops that penetrate the spinal cord parenchyma are best left truncated at the pial surface, avoiding serious injury. Partial resection of spinal AVMs has the benefit of addressing symptoms while also minimizing surgical morbidity [41]. Indocyanine-green videoangiography is routinely utilized for visualization of the arterialized or draining veins and confirmation of total excision [41].

\section{Postoperative period}

Patients are more likely to experience complications due to increased disease severity on presentation when compared with patients with less severe symptomology. Given that the presence of complications significantly increase the cost burden of spinal AVM treatment by more than US $\$ 30,000$, a discussion with respect to healthcare costs weighed against potential benefits following surgery should be established [42]. In addition, patients presenting with primarily motor deficits are more likely to improve following treatment, with sensory and sphincter disturbances showing less improvement [34]. Similar to CMs, inpatient rehabilitation may be necessary depending on the patients symptomlogy on presentation. Vertebral body bracing, heat, cold, and electrical stimulation are all potential avenues of pain management.

\section{Recommendations}

Similar to CMs, an ERAS protocol for spinal vascular malformations should prioritize preadmission counseling with respect to the possibility of neurologic worsening following intervention. Despite dural AVFs being more common and more likely than AVMs to result in transient decline, we believe patients with both lesion types should be briefed on the risks based on the present data available. In AVMs, lesion location is a factor implicated in patient outcomes, suggesting patient counseling should be tailored accordingly based on the lesion site. Counseling should also include a discussion on potential cost burden, with patients presenting with increased severity being more likely to incur higher costs due to increased risk of complications. The heterogeneous nature of spinal vascular malformations makes establishing a standard intraoperative ERAS paradigm difficult, with the decision to employ surgery or embolization being determined on a case-by-case basis. While effective as a stand-alone treatment, the relatively high lesion recurrence rate of embolization outweighs the benefits incurred from reducing complications related to surgery. The authors recommend a combined approach, with embolization being performed preoperatively followed by surgical resection (class IIa; level of evidence: C). GTR is preferred unless there is excessive parenchymal involvement. We recommend utilizing 3D-RA to better visualize complex angioarchitecture, aiding the neurosurgeon during resection. Following treatment, postoperative angiography and frequent follow-up is indicated to ensure recurrence does not occur, and we recommend follow-up imaging at 3 months (Fig. 2e) and 6 months (Fig. 2f), with continuing follow-up $1,3,5$, and 10 years post treatment. In addition, effective pain management and rehabilitation for persistent neurological sequela following surgery should be implemented. The factors discussed are all predictors of outcome that should be considered in developing an ERAS protocol for spinal cord surgery. 


\section{Intramedullary spinal cord tumors}

Intramedullary spinal cord tumors (IMSCTs) account for $2-4 \%$ of all CNS tumors, with ependymomas being the most common, followed by astrocytomas and other miscellaneous tumors (hemangioblastomas, gangliogliomas, germinomas, primary CNS lymphomas, melanomas, and rarely metastasis from primary malignancy) [43].

\section{Preoperative period}

Depending on the patients symptomology, detailed counseling outlining the risks of spinal cord surgery are necessary much like those described for the previous spinal cord pathologies. Asymptomatic lesions are better followed, especially those in eloquent regions, with patients who are steadily worsening being better candidates for surgery. Occurring most commonly in children, IMSCTs present with variable radicular or diffuse back pain that is worse at night, accompanied with scoliosis in $33 \%$ of patients. Somatosensory and motor loss are often misinterpreted as clumsiness in children, contributing to delayed diagnosis. Bladder and bowel dysfunction manifest in late-stage disease [43]. Surgical resection remains the primary treatment modality, followed by radiotherapy and chemotherapy.

\section{Intraoperative period}

Comprising $50-60 \%$ of all IMSCTs, ependymomas arise from ependymal cells lining the ventricles or central canal of the spinal cord. Despite being unencapsulated, ependymomas are well circumscribed with smooth, regular margins making GTR feasible and the first line of treatment (class I; level of evidence: C) [43, 44]. Ependymomas that are completely resected serve as a strong predictor of survival, with subtotal resection (STR) resulting in greater recurrence rates, although GTR has not been shown to impact functional outcome in patients [45, 46]. Given that myxopapillary ependymomas are derived from the filum terminale, they occur in the conus medularis, making GTR difficult. Adjuvant radiotherapy is considered a second line treatment (class IIa, level of evidence: C) following cases where only STR was possible [43]. Radiation myelopathy, impaired spine growth, radiation necrosis, vasculopathy, and a $25 \%$ risk of secondary tumors in 30 years are risk factors associated with radiotherapy treatment, suggesting its use should be avoided in children. For this reason, chemotherapy has been explored as a second line treatment (class IIb, level of evidence: C) following lesion resection in children [43].

Astrocytomas are another type of glioma and are the most common IMSCT in children [47]. They are classified into WHO Grade I-IV tumors, with the latter two grades being the most aggressive with a mean survival of 15.5 months. Symptoms of scoliosis, pain that is worse at night, abdominal pain, muscle wasting, and myelopathy are suggestive of astrocytoma, and most often present between 5 and 10 years of age [48]. Often times, diagnosis is delayed and incorrect workup is initiated in patients who present with nonspecific abdominal pain before motor symptom onset [49]. Surgery remains the primary treatment option (class IIb, level of evidence: C). Unlike ependymomas that have a clear plane of dissection, astrocytomas lack this plane and are more likely to infiltrate underlying spinal cord parenchyma, making GTR more difficult to achieve without causing injury. It is recommended that for higher grade tumors, the aim should be to achieve STR followed with adjuvant radiotherapy (class IIa, level of evidence: C) or chemotherapy (class IIb, level of evidence: C). In cases of tumor recurrence, radiotherapy is indicated, although reoperation has been reported successfully in the literature [50]. It should be noted that the following evidence recommendations are presented based on the findings of a single institution, nonsystematic review of spinal cord tumor surgery.

\section{Postoperative period}

Similar to other spinal cord tumors, functional outcome is largely dependent on the extent of neurological deficits prior to surgery. In IMSCTs, symptom duration of less than 2 years is associated with better outcomes [46]. The primary cause of dysfunction in IMSCTs is spinal cord compression. The location of spinal cord compression dictates symptom presentation, thus guiding the direction of postoperative rehabilitation. For example, upper motor neuron bowel dysfunction warrants the initiation of effective bowel evacuation program and pain prevention with defecation. Tumors causing sacral compression may result in bladder dysfunction, requiring hydronephrosis prevention, maintenance of bladder pressure, and urinary tract infection risk reduction. Depending on tumor location, postoperative rehabilitation efforts should be tailored accordingly [26].

\section{Recommendations}

Like other spinal cord pathologies, effective preoperative patient counseling is necessary to establish care goals early on in the treatment process. Patient's should be made aware of the potential for morbidity following surgical intervention, as well as the risks associated with abstaining from treatment. If the patient is asymptomatic, we recommend close monitoring with neuroimaging. Any evidence of new symptoms prompts rapid neurological workup. Patients already presenting with symptoms are advised to undergo resection, as waiting for disease progression and 
symptomatic worsening lends itself to poor functional recovery. To date, microsurgery is the preferred treatment modality for both ependymomas and astrocytomas, with radiotherapy generally being used in refractory cases. The role of chemotherapy in the treatment of gliomas appears promising, but more research is required. More studies assessing surgical intervention, radiotherapy, and chemotherapy are necessary to gain a better understanding of the role each modality can play in treating gliomas and their ultimate incorporation into an ERAS protocol. The postoperative period should focus on designing a rehabilitation program tailored to the needs of the patient. This includes paying close attention to the desired outcome of rehabilitation as defined by the patient, and setting appropriate expectations for recovery early on.

\section{Current state of ERAS and spinal cord surgery}

To date, only a single enhanced recovery after spine surgery (ERSS) study for 97 patients (41 ERSS and 56 pre-ERSS) undergoing spine surgery for spinal cord tumors has been reported; although, this study includes only metastatic tumors. Given that the majority of metastatic spinal tumors are osseous in nature, this study does not encompass primary spinal lesions, where there is still a lack of studies assessing ERAS in this domain. When comparing the preERSS and ERSS cohorts, the pre-ERSS group had a slightly longer hospital length of stay $(6.8 \pm 1.9$ days $)$ versus the ERSS group $(6.3 \pm 2.2$ days). The 30 -day readmission and 30-day complication rates were both lower in pre-ERSS patients $(8.9 \%$ vs $14.6 \% ; 17.9 \%$ vs $31.7 \%)$. Despite these differences, no statistical significance was reached for these end points $(p=0.590, p=0.381$, and $p=0.113$, respectively). When comparing pain, the ERSS group had lower average pain scores than the pre-ERSS group, although this did not reach statistical significance either.

It should be noted that intramedullary metastases are therapeutically challenging lesions, with surgical resection, radiotherapy, and chemotherapy having limited efficacy. In addition, drawing conclusions in this cohort of patients is problematic, as any observations made are confounded by comorbidities inherent of the primary oncologic disease.

Rehabilitation is also complicated in patients with lesions of metastatic potential, as treatment modalities that increase blood flow may promote tumor seeding [26]. Accommodations for cancer-related disorders such as cachexia, fatigue and psychological factors must all be made [26]. This contrasts with primary spinal cord tumors, which therefore provide a better conduit in assessing the effectiveness of ERAS protocols in spinal cord surgery.

\section{Conclusions}

In this review, the authors describe the nuances and current practices of intramedullary and extramedullary spinal cord surgery. Depending on the lesion, different procedures are employed that contribute to changes in patient outcome. While much has improved in the last decade, spinal cord surgery remains a challenging front for neurosurgeons and spine surgeons given the rarity of lesions and eloquent anatomy involved. Nevertheless, evidence-based practices that improve patient outcomes are beginning to emerge in literature. Although primarily in the form of nonrandomized, retrospective series, these new data provide a basis for which ERAS can be built off with respect to spinal cord surgery. In the future, rigorous randomized controlled trials will be required to establish efficacy for ERAS protocols in intramedullary and extramedullary spinal cord surgery. For now, the recommendations made in this review serve to establish a framework for which future ERAS studies can base their protocols on. Given the nature of ERAS programs as quality improvement initiatives, continuous data-driven reassessment and optimization will be necessary to ensure excellent outcomes following spinal cord surgery.

Author contributions SS was responsible for conception and design of the article, conducting the search, screening eligible studies, extracting data, interpreting results, and writing the article. ME was responsible for conducting the search, screening eligible studies, extracting data, interpreting results, and contributed to writing the report. PP was responsible for conducting the search, screening eligible studies, extracting data, and contributed to writing the report. PN contributed to screening eligible studies, interpreting results and provided feedback on the report. DMR was responsible for collecting data, creating both Figs. 1 and 2, and providing feedback on the report. JDS, MSP, and PT assisted with interpreting results and provided feedback on the article. MYSK was responsible for oversight of the article, design of the article, interpretation of results, and provided feedback on the report. All authors approved the final manuscript.

\section{Compliance with ethical standards}

Conflict of interest The authors declare that they have no conflict of interest.

Publisher's note: Springer Nature remains neutral with regard to jurisdictional claims in published maps and institutional affiliations.

\section{References}

1. Ljungqvist $\mathrm{O}$, Scott M, Fearon KC. Enhanced recovery after surgery. JAMA Surg. 2017;152:292.

2. Engelman RM, Rousou JA, Flack JE, Deaton DW, Humphrey $\mathrm{CB}$, Ellison LH, et al. Fast-track recovery of the coronary bypass patient. Ann Thorac Surg. 1994;58:1742-6.

3. Grasu RM, Cata JP, Dang AQ, Tatsui CE, Rhines LD, Hagan KB, et al. Implementation of an enhanced recovery after spine surgery 
program at a large cancer center: a preliminary analysis. J Neurosurg Spine. 2018;29:588-98.

4. Liang J, Bao Y, Zhang H, Huo L, Wang Z, Ling F. Management and prognosis of symptomatic patients with intramedullary spinal cord cavernoma. J Neurosurg Spine. 2011;15:447-56.

5. Pagni CA, Canavero S, Forni M. Report of a cavernoma of the cauda equina and review of the literature. Surg Neurol. 1990;33:124-31.

6. Padovani R, Tognetti F, Proietti D, Pozzati E, Servadei F. Extrathecal cavernous hemangioma. Surg Neurol. 1982;18:463-5.

7. Shukla D, Rao VS, Rajesh A, Uppin MS, Purohit AK. Lumbar extradural dumbbell cavernous hemangioma: a rare lesion. J Neurosci Rural Pract. 2013;4:207-9.

8. Haasdijk RA, Cheng C, Maat-Kievit AJ, Duckers HJ. Cerebral cavernous malformations: from molecular pathogenesis to genetic counselling and clinical management. Eur J Hum Genet. 2012;20:134-40.

9. Badhiwala JH, Farrokhyar F, Alhazzani W, Yarascavitch B, Aref M, Algird A, et al. Surgical outcomes and natural history of intramedullary spinal cord cavernous malformations: a singlecenter series and meta-analysis of individual patient data. J Neurosurg Spine. 2014;21:662-76.

10. Cosgrove GR, Bertrand G, Fontaine S, Robitaille Y, Melanson D. Cavernous angiomas of the spinal cord. J Neurosurg. 1988;68:31-6.

11. Sandalcioglu IE, Wiedemayer H, Gasser T, Asgari S, Engelhorn $\mathrm{T}$, Stolke D. Intramedullary spinal cord cavernous malformations: clinical features and risk of hemorrhage. Neurosurg Rev. 2003;26:253-6.

12. Walcott BP, Choudhri O, Lawton MT. Brainstem cavernous malformations: natural history versus surgical management. J Clin Neurosci. 2016;32:164-5.

13. Li J, Chen G, Gu S, Liu X, Shou J, Gu W, et al. Surgical outcomes of spinal cord intramedullary cavernous malformation: a retrospective study of 83 patients in a single center over a 12-year period. World Neurosurg. 2018;118:e105-14.

14. Muramoto A, Imagama S, Ito Z, Ando K, Tauchi R, Matsumoto $\mathrm{T}$, et al. The cutoff amplitude of transcranial motor evoked potentials for transient postoperative motor deficits in intramedullary spinal cord tumor surgery. Spine. 2014;39:1086-94.

15. Saadeh YS, Smith BW, Joseph JR, Jaffer SY, Buckingham MJ, Oppenlander ME, et al. The impact of blood pressure management after spinal cord injury: a systematic review of the literature. Neurosurg Focus. 2017;43:E20.

16. Lunardi P, Acqui M, Ferrante L, Fortuna A. The role of intraoperative ultrasound imaging in the surgical removal of intramedullary cavernous angiomas. Neurosurgery. 1994;34:520-3. discussion 523

17. Bozinov O, Burkhardt J-K, Woernle CM, Hagel V, Ulrich NH, Krayenbühl $\mathrm{N}$, et al. Intra-operative high frequency ultrasound improves surgery of intramedullary cavernous malformations. Neurosurg Rev. 2012;35:269-75.

18. Vishteh AG, Spetzler RF. Radical excision of intramedullary cavernous angiomas. Neurosurgery. 1999;44:428.

19. Deshmukh VR, Albuquerque FC, Zabramski JM, Spetzler RF. Surgical management of cavernous malformations involving the cranial nerves. Neurosurgery. 2003;53:352-7.

20. Vishteh AG, Sankhla S, Anson JA, Zabramski JM, Spetzler RF. Surgical resection of intramedullary spinal cord cavernous malformations: delayed complications, long-term outcomes, and association with cryptic venous malformations. Neurosurgery. 1997;41:1094-100. discussion1100-1.

21. Ryan RW, Spetzler RF, Preul MC. Aura of technology and the cutting edge: a history of lasers in neurosurgery. Neurosurg Focus. 2009;27:E6
22. Choudhri O, Karamchandani J, Gooderham P, Steinberg GK. Flexible omnidirectional carbon dioxide laser as an effective tool for resection of brainstem, supratentorial, and intramedullary cavernous malformations. Oper Neurosurg. 2014;10:34-45.

23. Mitha AP, Turner JD, Abla AA, Vishteh AG, Spetzler RF. Outcomes following resection of intramedullary spinal cord cavernous malformations: a 25-year experience. J Neurosurg Spine. 2011;14:605-11.

24. Cristante L, Hans-Dietrich H. Radical excision of intramedullary cavernous angiomas. Neurosurgery. 1998;43:424-31.

25. McKinley WO, Huang ME, Tewksbury MA. Neoplastic vs. traumatic spinal cord injury: an inpatient rehabilitation comparison. Am J Phys Med Rehabil. 2000;79:138-44.

26. Raj VS, Lofton L. Rehabilitation and treatment of spinal cord tumors. J Spinal Cord Med. 2013;36:4-11.

27. Kim LJ, Spetzler RF. Classification and surgical management of spinal arteriovenous lesions: arteriovenous fistulae and arteriovenous malformations. Neurosurgery. 2006;59:S3-195-S3-201.

28. Krings T. Vascular malformations of the spine and spinal cord*. Clin Neuroradiol. 2010;20:5-24.

29. Patsalides A, Knopman J, Santillan A, Tsiouris AJ, Riina H, Gobin YP. Endovascular treatment of spinal arteriovenous lesions: beyond the dural fistula. https://doi.org/10.3174/ajnr.A2190

30. Rosenblum B, Oldfield EH, Doppman JL, Di Chiro G. Spinal arteriovenous malformations: a comparison of dural arteriovenous fistulas and intradural AVM's in 81 patients. J Neurosurg. 1987;67:795-802.

31. Runnels JB, Hanbery JW. Spontaneous subarachnoid hemorrhage associated with spinal cord tumor. J Neurosurg. 1974;40:252-4.

32. Rangel-Castilla L, Russin JJ, Zaidi HA, Martinez-del-Campo E, Park MS, Albuquerque FC, et al. Contemporary management of spinal AVFs and AVMs: lessons learned from 110 cases. Neurosurg Focus. 2014;37:E14.

33. Wojciechowski J, Kunert P, Nowak A, Dziedzic T, Czernicki T, Wójtowicz K, et al. Surgical treatment for spinal dural arteriovenous fistulas: outcome, complications and prognostic factors. Neurol Neurochir Pol. 2017;51:446-53.

34. Cenzato M, Debernardi A, Stefini R, D'Aliberti G, Piparo M, Talamonti G, et al. Spinal dural arteriovenous fistulas: outcome and prognostic factors. Neurosurg Focus. 2012;32:E11.

35. Kalani MYS, Ahmed AS, Martirosyan NL, Cronk K, Moon K, Albuquerque FC, et al. Surgical and endovascular treatment of pediatric spinal arteriovenous malformations. World Neurosurg. 2012;78:348-54.

36. Ozpeynirci Y, Schmitz B, Schick M, Konig R. Role of threedimensional rotational angiography in the treatment of spinal dural arteriovenous fistulas. Cureus. 2017;9:e1932.

37. Kang MS, Kim KH, Park JY, Kuh SU, Chin DK, Jin BH, et al. Comparison of endovascular embolization and surgery in the treatment of spinal intradural dorsal arteriovenous fistulae. World Neurosurg. 2018. https://doi.org/10.1016/j.wneu.2018. 11.093

38. Česák T, Adamkov J, Poczos P, Kanta M, Krajina A, Krajíčková $\mathrm{D}$, et al. Multidisciplinary approach in the treatment of spinal dural arteriovenous fistula-results of endovascular and surgical treatment. Acta Neurochir. 2018;160:2439-48.

39. Gokhale S, Khan SA, McDonagh DL, Britz G. Comparison of surgical and endovascular approach in management of spinal dural arteriovenous fistulas: a single center experience of 27 patients. Surg Neurol Int. 2014;5:7.

40. Niimi Y, Sala F, Deletis V, Berenstein A. Provocative testing for embolization of spinal cord AVMs. Inter Neuroradiol. 2000;6:191-4.

41. Kalani MYS, Ahmed AS, Martirosyan NL, Cronk K, Moon K, Albuquerque FC, et al. Surgical and endovascular treatment of 
pediatric spinal arteriovenous malformations. World Neurosurg. 2012;78:348-54.

42. Yue JK, Deng H, Winkler EA, Ordaz A, Gillis-Buck EM, Lee YM, et al. Hospital complications and costs of spinal arteriovenous malformations in the United States from 2002-14. J Neurosurg Sci. 2018. https://doi.org/10.23736/S0390-5616.18. 04552-6

43. Tobin MK, Geraghty JR, Engelhard HH, Linninger AA, Mehta AI. Intramedullary spinal cord tumors: a review of current and future treatment strategies. Neurosurg Focus. 2015;39:E14.

44. Kucia EJ, Bambakidis NC, Chang SW, Spetzler RF. Surgical technique and outcomes in the treatment of spinal cord ependymomas, part 1: intramedullary ependymomas. Oper Neurosurg. 2011;68:ons57-63.

45. Feldman WB, Clark AJ, Safaee M, Ames CP, Parsa AT. Tumor control after surgery for spinal myxopapillary ependymomas: distinct outcomes in adults versus children. J Neurosurg Spine. 2013;19:471-6.

46. Guidetti B, Mercuri S, Vagnozzi R. Long-term results of the surgical treatment of 129 intramedullary spinal gliomas. J Neurosurg. 1981;54:323-30.

47. Teng YD, Abd-El-Barr M, Wang L, Hajiali H, Wu L, Zafonte RD. Spinal cord astrocytomas: progresses in experimental and clinical investigations for developing recovery neurobiology-based novel therapies. Exp Neurol. 2019;311:135-47.

48. Jallo GI, Freed D, Epstein F. Intramedullary spinal cord tumors in children. Child's Nerv Syst. 2003;19:641-9.
49. Jackson K, Lapsia S, Strunc M, Tye G. Spinal cord astrocytoma: a unique presentation of abdominal pain. Radio Case Rep 2018;13:284-8.

50. Mottl H, Koutecky J. Treatment of spinal cord tumors in children. Med Pedia Oncol. 1997;29:293-5.

51. Bian LG, Bertalanffy H, Sun QF, Shen J-K. Intramedullary cavernous malformations: clinical features and surgical technique via hemilaminectomy. Clin Neurol Neurosurg. 2009;111:511-7.

52. Fujiwara Y, Manabe H, Izumi B, Shima T, Adachi N. Remarkable efficacy of temozolomide for relapsed spinal myxopapillary ependymoma with multiple recurrence and cerebrospinal dissemination: a case report and literature review. Eur Spine J. 2018;27:421-5.

53. Jallo GI, Freed D, Zareck M, Epstein F, Kothbauer KF. Clinical presentation and optimal management for intramedullary cavernous malformations. Neurosurg Focus. 2006;21:e10.

54. Kim LJ, Klopfenstein JD, Zabramski JM, Sonntag VKH, Spetzler RF. Analysis of pain resolution after surgical resection of intramedullary spinal cord cavernous malformations. Neurosurgery. 2006;58:106-11.

55. Kim W-H, Yoon SH, Kim C-Y, Kim K, Lee MM, Choe G, et al Temozolomide for malignant primary spinal cord glioma: an experience of six cases and a literature review. J Neurooncol. 2011;101:247-54.

56. Zevgaridis D, Medele RJ, Hamburger C, Steiger HJ, Reulen HJ. Cavernous haemangiomas of the spinal cord. A review of 117 cases. Acta Neurochir. 1999;141:237-45. 